J. Clin. Chem. Clin. Biochem.

Vol. 15,1977, pp. 289-291

\title{
Verwendung von Primaten-Gewebshomogenaten zur Qualitätskontrolle der Bestimmung von Kreatin- kinase-MB mit inhibierenden Antikörpern
}

\author{
Von D. Neumeier, Barbara Siegele, Barbara Glück und M. Knedel \\ Institut für Klinische Chemie am Klinikum Großhadern der Ludwig-Maximilians-Universität München
}

(Eingegangen am 29. Oktober/16. Dezember 1976)

Zusammenfassung: Nach chromatographischer Trennung der Kreatinkinase-Isoenzyme CK-MM,CK-MB und CK-BB aus Gewebshomogenaten von Rhesusaffen konnte gezeigt werden, daß die Aktivität der Untereinheit CK-M durch inhibitorisches Anti-CK-M-Serum vollständig gehemmt wird. Damit sind Kontrollproben aus Affengewebe prinzipiell zur Qualitätskontrolle der immunologischen Bestimmung von Kreatinkinase-MB geeignet. Präzision in der Serie: $\mathrm{n}=56, \overline{\mathrm{x}}=29,2 \mathrm{U} / 1, \mathrm{~s}=3,2 \mathrm{U} / \mathrm{l}, \mathrm{VK}=11,1 \%$, Präzision von Tag zu Tag: $\mathrm{n}=12, \overline{\mathrm{x}}=166,7 \mathrm{U} / 1, \mathrm{~s}=5,0 \mathrm{U} / 1$, $\mathrm{VK}=3,0 \%$. Die Entwicklung von Kontrollseren mit katalytischen Konzentrationen von Kreatinkinase-MB im unteren Konzentrationsbereich ist notwendig.

The use of primate tissue homogenates for quality control in the creatine kinase-MB determination with inhibiting antibodies

Summary: The creatine kinase isoenzymes CK-MM, CK-MB and CK-BB from Rhesus monkey heart and brain tissue homogenates were separated chromatographically. Thereafter it could be demonstrated that the activity of the simian subunit CK-M is completely inhibited by anti-inhibitory-CK-M serum. Thus control sera from simian tissue are in principle suited for quality control in an immunological determination of creatine kinase-MB. The intraassay variance and interassay variance were $n=56, \bar{x}=29.2 \mathrm{U} / 1, \mathrm{SD}=3.2 \mathrm{U} / 1, \mathrm{CV}=11.1 \%$ and $n=12, \bar{x}=166.7 \mathrm{U} / 1$, $\mathrm{SD}=5.0 \mathrm{U} / 1, \mathrm{CV}=3.0 \%$ respectively. It is desirable to develop control sera with catalytic concentrations of creatine kinase-MB in a lower range.

\section{Einleitung}

In den beiden letżten Jahren hat die Bestimmung der Isoenzyme der Kreatinkinase (ATP: Creatin-Phosphotransferase (CK) EC 2.7.3.2) wegen ihrer unterschiedlichen Organverteilung (1) zunehmend an differentialdiagnostischer Bedeutung gewonnen. Durch die Einführung von Techniken, die sich chromatographischer (2) und immunologischer Trennschritte $(3-6)$ bedienen, wurde die quantitative Bestimmung wesentlich vereinfacht und beschleunigt. Die Bestimmung der Isoenżyme, insbesondere des Isoenzyms CK-MB ist somit auch in der klinịsch-chemischen Notfalldiagnostik möglich. Die quantitative Bestimmung und der Anteil von Kreatinkinasse-MB hat für die differentialdiagnostische Abklärung und die Verlaufsbeobachtung des Myokardinfarktes große klinische Bedeutung. Wegen der diagnostischen Konsequenzen und der zunehmenden Untersuchungszahlen muß eine ausreichende Qualitätskontrölle der Isoenzymbestimmung gewährleistet sein. Wir berichten über vorlậufige Ėrgebnisse zur Qualitätskontrolle bei
Verwendung von heterologen Herzmuskelhomogenaten zur immunologischen Bestimmung von KreatinkinaseMB mit inhibierenden Antikörpem.

\section{Material und Methoden}

Als Kontrolle verwendeten wir Herzmuskelhomogenate von Rhesus-Affen ${ }^{1}$ ), die in 1-ml-Portionen lyophilisiert waren (7). Nach Rekonstitution und chromatographischer Trennung (2) der Homogenate $(n=5)$ fanden wir für die einzelnen Isoenzyme folgende katalytische Konzentrationen: CK-MM 381,7 \pm 19 U/1 (Peak 1), CK-MB $42 \pm 18$ U/1 (Peak 2), CK-BB $114 \pm 25$ U/1 (Peak 3). Die Bestimmung der Kreatinkinase-B erfolgte mit der von uns mitgeteilten Methode $(5,6,8)$ unter Verwendung von 1-Test-Präparationen (Merck-1-Test CK-MB) in Halbmikroansätzen. Zur Präzisionskontrolle von Tag zu Tag wurden jeweils frisch rekonstituierte Homogenatpräparationen verwendet. $\mathrm{Nach}$ Abzug eines Reagenzienleerwertes wurde die Intra- und Interassayvarianz in verschiedenen Konzentrationsbereichen berechnet. Daneben wurde die Haltbarkeit der Homogenate nach Rekonstitưtion untersucht. Zur Spezifitätskontrolle der Methode im

1) CPK-Isoenzym-Markierungsserum, Merz u. Dade GmbH, D-8000 München 50, Lerchenstraße 5, Best.-Nr. B 5312-32. 
heterologen System überprüften wir nach chromatographischer Trennung der Affengeivebshomogenate die Hemmung der Kreatinkinase-Aktivität in den einzelnen Fraktionen nach Zugabe des inhibitorischen Anti-CK-M-Serums.

\section{Ergebnisse}

Für die Intraassayvarianz ergab sich: Verdünnung 1:5 mit inaktiviertem Serum $\mathrm{n}=56, \overline{\mathrm{x}}=29,2 \mathrm{U} / 1, \mathrm{~s}=3,2 \mathrm{U} / 1$, VK $=11,1 \%$; Verdünnung 1:10 mit inaktiviertem Serum $\mathrm{n}=34, \overline{\mathrm{x}}=10,1 \mathrm{U} / \mathrm{l}, \mathrm{s}=1,4 \mathrm{U} / 1, \mathrm{VK}=13,6 \%$. Entsprechend errechnete sich für die Interassayvarianz: $\mathrm{n}=12, \overline{\mathrm{x}}=166,7 \mathrm{U} / 1, \mathrm{~s}=5,0 \mathrm{U} / 1, \mathrm{VK}=3,0 \%$; Verdünnung $1: 5$ mit inaktiviertem Serum $n=12, \bar{x}=29,1 \mathrm{U} / 1$, $s=2,2 \mathrm{U} / \mathrm{l}, \mathrm{VK}=7,6 \%$.

Nach chromatographischer Trennung des Primatenhomogenates konnte durch Zugabe von inhibitorișchem Anti-CK-M-Serum im Rahmen des methodischen Fehlers die Aktivität der Kreatinkinase im ersten Peak (MM-Peak) zu 100\%, in den Fraktionen des zweiten Peaks (MB-Peak) zu 50\% und in den Fraktionen des dritten Peaks (BB-Peak) zu 0\% inhibiert werden. Nach Rekonstitution des lyophilisierten Primatenhomogenates konnte ohne Verdünnung bis zu 7 Stunden die Ausgangsaktivität für Kreatinkinase-B gemessen werden. Nach 24 Stunden waren noch $94 \%$ der Aktivität zu messen (Abb. 1). Bei Verdünnung mit inaktiviertem Serum zeigte sich bei der Bestimmung von Kreatinkinase-B innerhalb der 1. Stunde im Gegensatz zur Bestimmung der Gesamt-Kreatinkinase innerhalb der 1 . Stunde ein deutlicher Aktivitätsabfall.

\section{Diskussion}

Ebenso wie im homologen System (6) werden in dem hier verwendeten heterologen System nach chromatographischer Trennung und Zugabe des Antiserums die Aktivitäten der einzelnen Kreatinkinase-Fraktionen entsprechend ihrem Anteil an CK-M-Untereinheiten gehemmt. Durch die vollständige Inhibition der CK-MUntereinheit wird die Spezifität des CK-M-Serums auch für das Primaten-Isoenzym bestätigt (8). Die geringen Speziesunterschiede der einzelnen Isoenzyme der Kreatinkinase ließen dieses Ergebnis vermuten.

Die Ergebnisse der Präzịisionskontrollversuche im heterologen System liegen für verschiedene Konzentrationsbereiche nur geringfügig unter den Ergebnissen, die bei Verwendung gepoolter Seren von Patienten mit Herzinfarkt oder humaner Herzmuskelhomogenate erhalten wurden $(6,9)$. In der Interassayvarianz muß wegen jeweils neu anzusetzender Verdünnungen einerseits ein differierender Verdünnungsfehler berücksichtigt werden, andererseits zeigten die einzelnen von uns verwendeten Präparationen dès Isoenzym-Markèrs relativ hohe Aktivitätsschwankungen. Wegen dieser präparativen Schwankungen und des Fehlens einer anerkannten Referenzmethode für die Bestimmung der Isoenzyme der Kreatinkinase ist eine Richtigkeitskontrolle nur bedingt möglich.

Zur Präzisionskontrolle scheint uns eine Verdünnung des Primatenhomogenates, das kommerziell zur Quali-
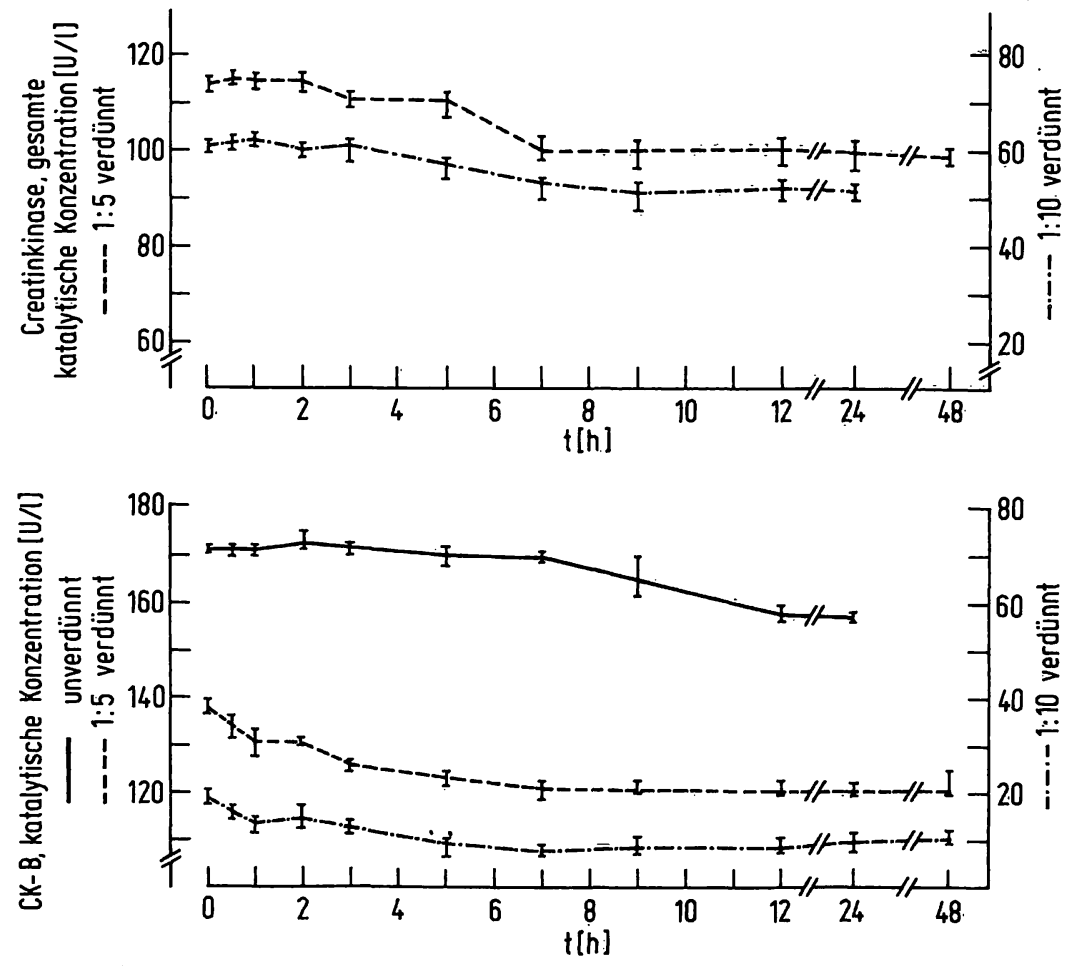

Abb. 1. Untersuchung der Stabilität von Kreatinkinase-(Gesamt) und Kreatinkinase-MB in den rekonstituierten Affengewebshomogenaten: unverdünnt $\longrightarrow, 1: 5$ mit inaktiviertem Serum verdünnt $--,, 1: 10$ mit inaktiviertem Serum verdünnt -...-. Während für die Aktivität der Gesamt-Kreatinkinase über 24 Stunden relativ konstant bleibt, kommt es bei Bestimmung der Kreatinkinase-B-Untereinheit in verdünnten Präparationen zu einem Aktivitätsabfall. 
tätskontrolle der elektrophoretischen Trennung der Isoenzyme erhältlich ist, sinnvoll, da mit der immunologischen Inhibitionsmethode gegenüber der Elektrophorese niedrigere Aktivitäten von Kreatinkinase-MB erfaßt werden können. Die gesicherte Bestimmung von Kreatinkinase-MB ist aber im unteren Konzentrationsbereich, der für die Frühdiagnose des Myokardinfarktes von Bedeutung ist, notwendig.

Prinzipiell ist das Primatenhomogenat zur routinemäßigen Qualitätskontrolle der Bestimmung von Kreatinkinase-

\section{Literatur}

1. Roberts, R., Henry, P. D. \& Sobel, B. E. (1975), Circulation $52,743-754$

2. Mercer, D. W. (1974), Clin. Chem. 20, 36-40.

3. Jockers-Wretou, E., Grabert, K. \& Pfleiderer, G. (1975), dieses J. 13, 85-88.

4. Neumeier, D., Knedel, M., Würzburg, U., Hennrich, N. \& Lang, H. (1975), Klin. Wochenschr. 53, 329-333.

5. Würzburg, U., Hennrich, N., Lang, H., Prellwitz, W., Neumeier, D. \& Knedel, M. (1976), Klin. Wochenschr. 54, $357-360$.
MB bei Verwendung der immunologischen Inhibitionsmethode geeignet. Nach den Angaben des Herstellers ist das Material nach Überprüfung mit den üblichen Methoden nicht reaktiv für $\mathrm{HB}_{\mathrm{s}} \mathrm{Ag}$, so daß die Gefahr einer Hepatitis-Infektion ausgeschlossen werden kann. Die arbeitsintensive Herstellung von Poolserum mit Kreatinkinase-MB-Aktivität oder humanen Herzmuskelhomogenaten zur Präzisionskontrolle ist somit nicht notwendig. Zur Richtigkeitskontrolle wäre eine Präparation mit erheblich geringerer Schwankungsbreite der Aktivität wünschenswert.
6. Neumeier, D., Prellwitz, W., Würzburg, U., Brundobler, M., Olbermann, M., Just, H.-J., Knedel, M. \& Lang, H. (1976), Clin. Chim. Acta 73, 445-451.

7. Welsh, M. \& Srebro, S. (1975), Clin. Chem. 21, 963.

8. Würzburg, U., Hennrich, N., Orth, H.-D., Lang, H., Prellwitz, W., Neumeier, D., Knedel, M. \& Rick, W. (1977), dieses J. 15, 131-137.

9. Prellwitz, W., Neumeier, D., Knedel, M., Lang, H., Würzburg, U., Schönborn, H. \& Schuster, P. H. (1976), Dtsch. Med. Wochenschr. 101, 983-988. 
